Bull. Mater. Sci., Vol. 13, No. 3, June 1990, pp. 197-204. (C) Printed in India.

\title{
Electrical conduction of sodium acetylacetonate
}

\author{
A SAWABY \\ Physics Department, National Research Centre, Dokki, Cairo, Egypt \\ MS received 5 August 1989; revised 30 November 1989
}

\begin{abstract}
The temperature dependence of DC electrical conductivity $\sigma$ of sodium acetylacetonate compound $\mathrm{Na}(\mathrm{ac}, \mathrm{ac})$ is studied for fresh and polarized samples. Both samples show semiconductor behaviour, but the value of $\sigma$ for the fresh sample is higher than that of the polarized sample. Three distinct regions are observed in the temperature conductivity curves. The conduction mechanism of each region is discussed. Anomalous conductivity phenomena are observed above $105^{\circ} \mathrm{C}$ and attributed to unidirectional intermolecular or phase transformation. The results are discussed on the basis of ionic conduction and some conduction parameters such as conduction energy $E_{c}$, transport energy $E_{u}$, ion mobility $u$, free ion density $n$, are calculated.
\end{abstract}

Keywords. Activation energy; electrical conductivity; phase transformation; sodium acetylacetonate.

\section{Introduction}

Metal acetylacetonate compounds $\mathrm{M}(\mathrm{ac}, \mathrm{ac}) x$ are of increasing importance in many applied and industrial scientific fields. They are mostly used as catalysts or chemical activators. A number of investigations (Kamel et al 1975; Amin et al 1980; Sahai and Verma 1981; Kadry et al 1984; Molokhia 1986) has been made for some of these compounds to determine their electrical properties such as dielectric constant, dielectric loss, pyroelectric behaviour and electrical conductivity, but the available data is still far from being complete.

The aim of the present work is to study the temperature dependence of DC electrical conductivity of one of the most important metal acetylacetonate compounds which is sodium acetylacetonate $\mathrm{Na}(\mathrm{ac}, \mathrm{ac})$, to collect more data and knowledge about the possible phenomena and the conduction behaviour of this compound.

\section{Experimental technique}

Sodium acetylacetonate compound $\mathrm{Na}(\mathrm{ac}, \mathrm{ac})$ was prepared according to standard procedures given in the literature (Fernelius 1949; Lowis et al 1948). Recrystallization of the products was carried out in ethyl-alcohol and the samples were dried under vacuum. The purity of the sample was tested by measurement of melting point $\left(203^{\circ} \mathrm{C}\right)$ and infrared spectra which agreed well with the reported values in the literature. For the electrical conductivity measurements, equal weights of the samples in powder form were placed in a special die. The die is placed in hydraulic press for $15 \mathrm{~min}$ under $10,000 \mathrm{~kg}$ in vacuum (Beckman 00-25 Glenrothes, Fife, Scotland), to form a disc $1.2 \mathrm{~cm}$ in diameter and $1.7 \mathrm{~mm}$ thick. The disc was sandwiched between two optical quality surfaces of a copper electrode using a specially designed holder to eliminate contact resistance. The $I-V$ characteristics 
indicate that the assembly of sample and electrodes is ohmic in character (Sawaby et al, unpublished).

A high impedence electrometer (VA-J 52, Dresden DDR) and a multiflex Galvanometer Type MG2 were used through a conventional electrical circuit. The measurements were carried out in a dry atmosphere keeping all experimental parameters unaltered in order to obtain comparable values.

Polarization of samples was carried out by first heating them to a specific polarizing temperature $T_{p}$ and then applying an electric polarizing field $E_{p}$ for a known polarizing time $t_{p}$. Subsequently, the samples were cooled to room temperature under the applied field and the field then reduced to zero.

\section{Results}

It was noticed that the time dependence of the current at voltages less than $12 \mathrm{~V} \mathrm{~cm}^{-1}$ is negligible. Therefore, to avoid the polarization effect on the conductivity behaviour of $\mathrm{Na}(\mathrm{ac}, \mathrm{ac})$ sample, field strengths of value less than $12 \mathrm{~V} \mathrm{~cm}^{-1}$ should be used.

Figure 1 shows the relation between $\log \sigma$ and reciprocal absolute temperature over the temperature $\left(20-160^{\circ} \mathrm{C}\right)$, for fresh and polarized $\mathrm{Na}(\mathrm{ac}, \mathrm{ac})$ samples. For the heating curves, it can be seen from this figure that the electrical conductivity $\sigma$ of fresh sample is higher than that of the polarized sample. However, the curve can be divided into three regions-the first region from 20 to $105^{\circ} \mathrm{C}$, the second region from 105 to $125^{\circ} \mathrm{C}$ and the third region from 125 to $160^{\circ} \mathrm{C}$. For the first region, it was noticed that the relation between $\log \sigma$ and $10^{3} / T$ gives straight parallel lines for fresh and polarized samples, i.e. obeys the well-known Arrhenius relation:

$$
\sigma=\sigma_{0} \exp \left[-E_{c} / k T\right]
$$

where $\sigma$ is the conductivity corresponding to a temperature $T$ and $E_{c}$ is the apparent activation energy, $\sigma_{0}$ is a constant and $k$ is the Boltzman's constant. The energy of the conduction $E_{c}$ was calculated from the slope of the straight line of the first region and is found to be $1.68 \mathrm{eV}$. For the second region, slight decrease in the conductivity values are observed by increasing the temperature, followed by fast decay in the conductivity curve at the third region for both samples. On the other hand, it is also noticed that the values of $\sigma$ are strictly reproducible at all temperatures of the first region for both samples, while the reproducibility of $\sigma$ is not indicated for the second and third regions. However, as the sample is taken over the heating-cooling cycle from the first region to the second region, the break-point between the two regions appears only during the heating process. On the contrary, the reproducibility of $\sigma$ is greatly impaired and the break-point between the first and second regions disappears completely during the heatingcooling cycle from the first to the third region and vice versa as shown in figure 1 . The irreproducibility and decay process of $\sigma$ which take place at the second and third regions may be attributed to possible existence of an irreversible intermolecular change starting slowly at the second region. Therefore it can be considered that this region is the transition region between the two straight lines of the first and third regions. However little inference can be drawn from figure 1. Therefore, for complete identification of the anomalous phenomena, several 


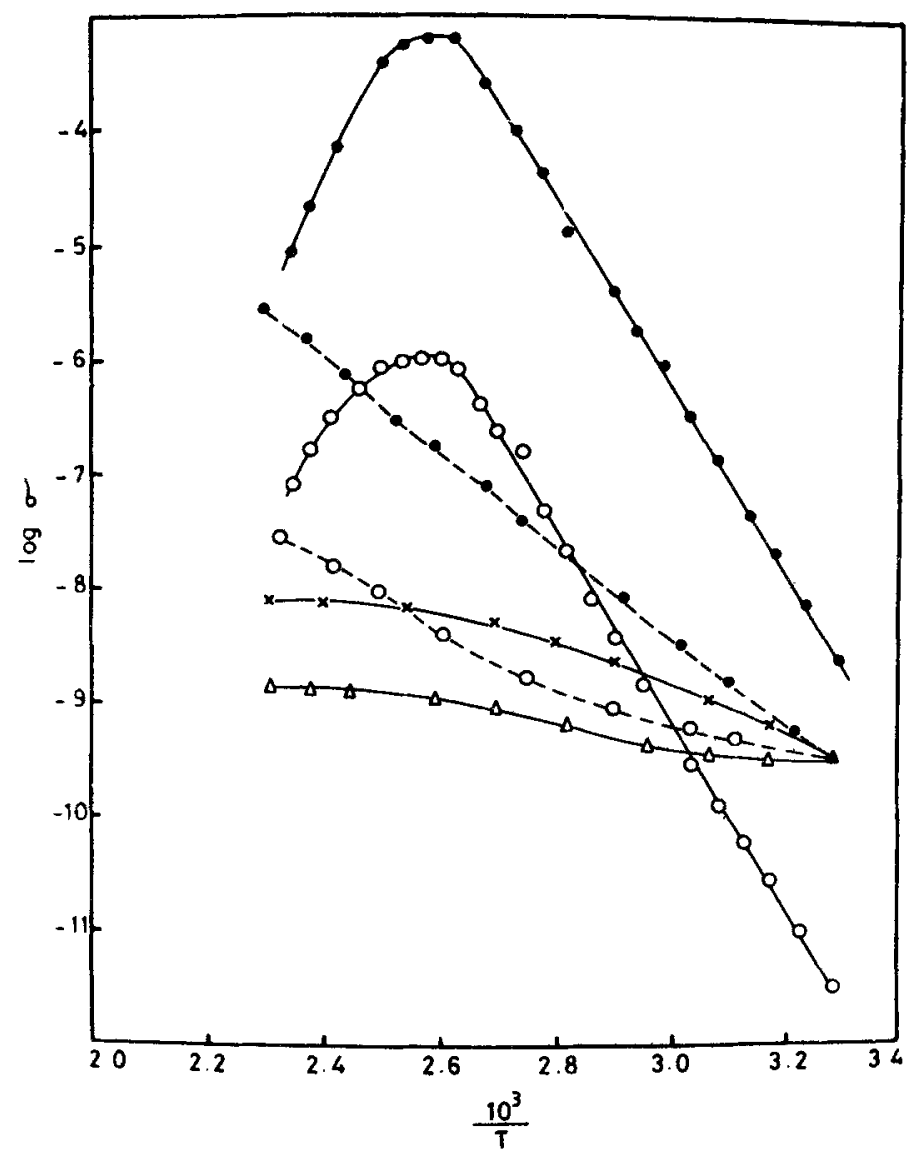

Figure 1. The electrical conductivity of $\mathrm{Na}(\mathrm{ac}, \mathrm{ac})$ as a function of the reciprocal temperature. Solid line for heating, dotted line for cooling $(\odot)$ fresh sample; $(O)$ polarized sample, $T_{p}=60^{\circ} \mathrm{C}, E_{p}=30 \mathrm{~V} \mathrm{~cm}^{-1}, t_{p}=30 \mathrm{~min} ;(\times)$ reheating the unpolarized sample; $(\Delta)$ reheating the polarized sample.

experimental runs were carried out at different temperatures corresponding to the transition region $\left(105,110,115,120\right.$ and $\left.125^{\circ} \mathrm{C}\right)$, to study the isothermal annealing effect on the conduction current of $\mathrm{Na}(\mathrm{ac}, \mathrm{ac})$ for different applied fields. Figure 2 represents one of these experiments at $115^{\circ} \mathrm{C}$. It can be seen from this figure that the time dependence of the current at an applied field of $10 \mathrm{~V} \mathrm{~cm}^{-1}$ is nearly negligible, but it becomes conspicuous at fields above $12 \mathrm{~V} \mathrm{~cm}^{-1}$ and the gradient tends to increase with the applied field. Similar plots were made at $105,110,120$ and $125^{\circ} \mathrm{C}$ but are not presented here. It was also noticed that the decay in the conductivity at this region not only depends on the applied field but is also affected by the annealing temperature. Therefore, the isothermal annealing effect was also studied at different annealing temperatures for a constant applied field strength of $30 \mathrm{~V} \mathrm{~cm}^{-1}$ as shown in figure 3 . It can be seen from this figure that the values of the current density generally decrease with heating time. At higher temperatures, shorter heating periods are needed to reach a minimum value of the current density which reflects the temperature dependence of the current decay. 


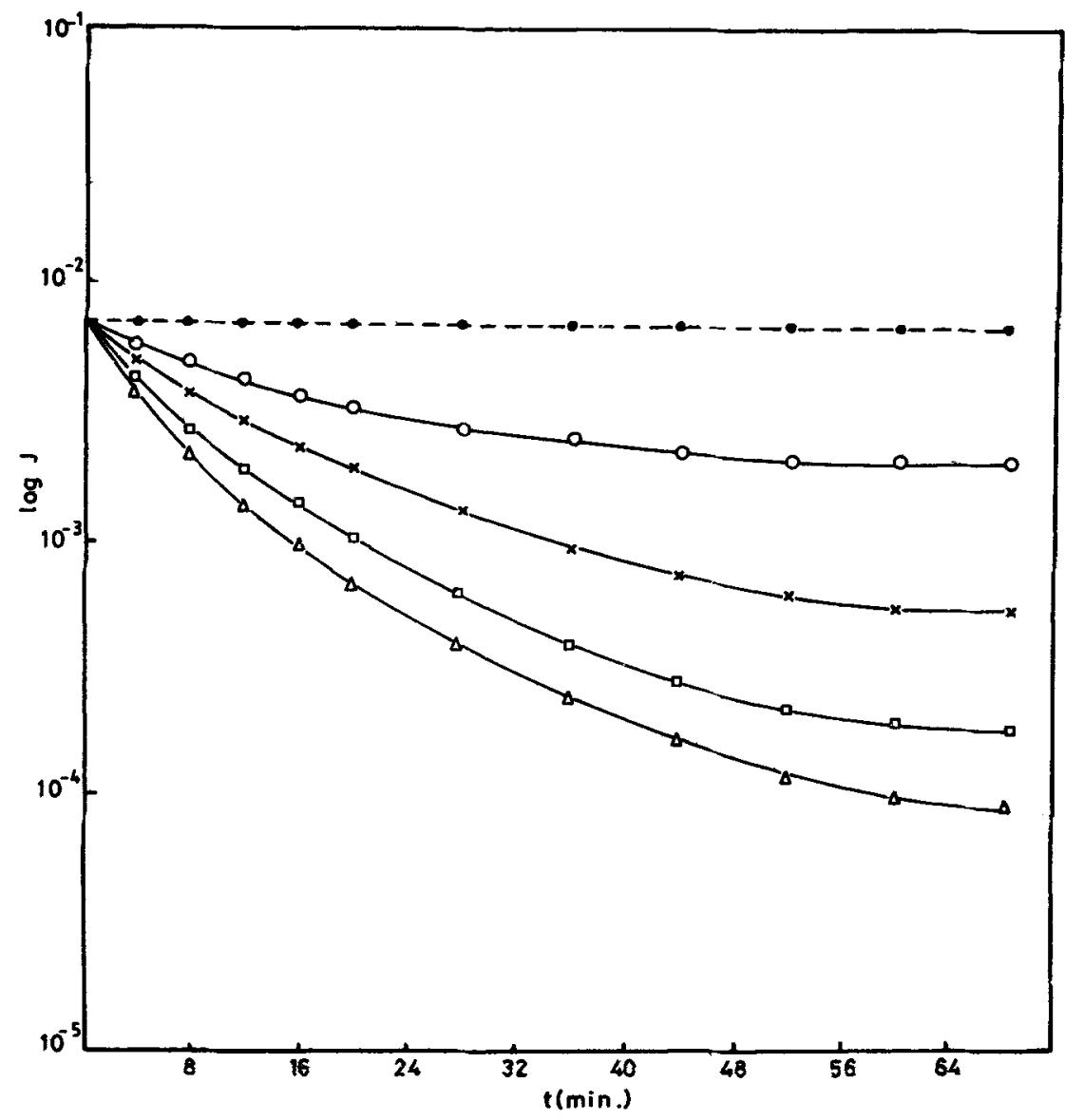

Figure 2. Isothermal annealing curves at different applied field (log $J$ against time) at $115^{\circ} \mathrm{C}$ for applied field of: $(O) 10 ;(O) 15 ;(\times) 20 ;(\square) 30 ;(\Delta) 40 \mathrm{~V} \mathrm{~cm}^{-1}$.

\section{Discussion}

The temperature dependence of the electrical conductivity $\sigma$ of $\mathrm{Na}(\mathrm{ac}, \mathrm{ac})$ shows semiconductor behaviour, when the values of the specific conductivity (which was measured under the present experimental conditions) are increased from $10^{-9} \mathrm{ohm}^{-1} \mathrm{~cm}^{-1}$ at room temperature to about $10^{-4} \mathrm{ohm}^{-1} \mathrm{~cm}^{-1}$ at $105^{\circ} \mathrm{C}$ as seen in figure 1. The apparent activation energy calculated from the straight line of the first region is $1.68 \mathrm{eV}$. It is sometimes assumed, as a general rule, that values for activation energies greater than 0.6 or $0.8 \mathrm{eV}$ would normally be associated with ionic transport and values less than $0.2 \mathrm{eV}$ should be considered as indicating undoubtedly electronic mechanism (Jonscher 1967; Jain et al 1978). Therefore the value of $1.68 \mathrm{eV}$ may be taken in favour of an ionic conduction mechanism operating in the temperature range corresponding to the first region. On the other hand, the irreproducibility of $\sigma$ observed at the second and the third regions of figure 1, may be attributed to possible existence of an irreversible phase 


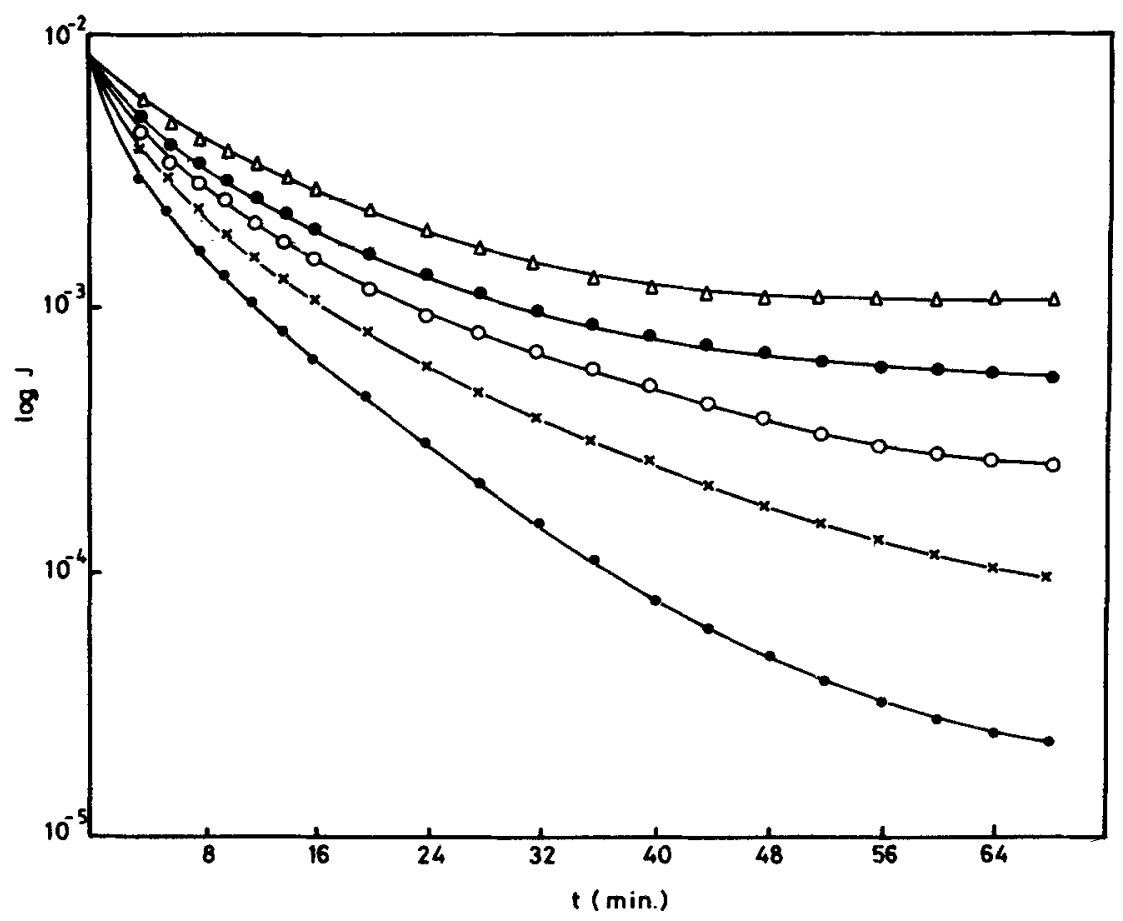

Figure 3. Isothermal annealing curves at different temperature and constant applied field of $30 \mathrm{~V} \mathrm{~cm}^{-1}\left(\log J\right.$ against time) $(\triangle) 105,(\odot) 110,(\bigcirc) 115,(\times) 120,(\cdot) 125^{\circ} \mathrm{C}$.

transiormation starting slowly in the transition region (second region) and completely altered in the third region.

The isothermal annealing study shows that the observed change is thermally and electrically activated.

It will be assumed here to a first approximation that the process in action is controlled by a chemical rate equation of type (Kamel and Attia 1961),

$$
t \exp (-\triangle E / k T)=\text { constant, }
$$

where $k$ is the Boltzman's constant, $t$ the time of change and $\triangle E$ the energy activating the process. The equivalent times and temperatures prescribing a particular degree of change were measured by the cross-cut method from the regular part of the isothermal annealing curves given in figure 3 . The activation energy $\triangle E$ for this process was deduced from (2) by plotting the logarithm of the time versus the reciprocal of the absolute temperature. Figure 4 shows a parallel set of straight lines. The slopes of these lines give an average value of the $\Delta E$ amounting to $0.82 \mathrm{eV}$. On the other hand, more information about the current decay phenomena could be obtained by applying the following equations (Masamitsu et al 1970).

$$
J=J_{0} \exp \left[-\mu_{i} E t / l\right]
$$

where $J$ is the current density, $\mu_{i}$ the mobility, $l$ the thickness of the sample and $t$ the time. 


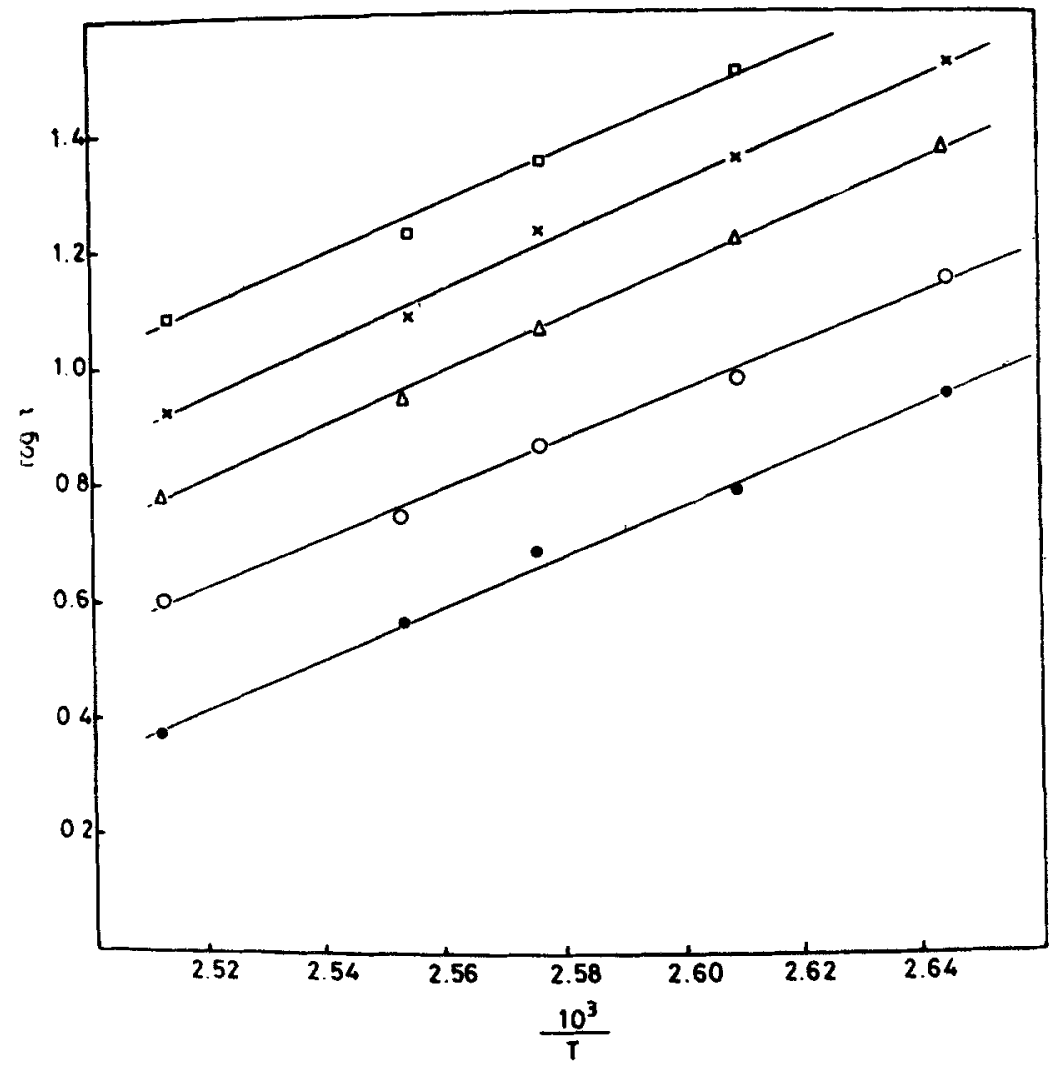

Figure 4. Equivalent time temperature curves for the observed decay in the current density during the isothermal annealing processes derived from figure 3.

$$
J_{0}=q M_{0} \mu E
$$

where $q$ is the ion charge, $E$ the electrical field and $M_{0}$ the density of the mobile ion at time $t=0$. However, the initial gradient of $\log I$ vs $t$ curves in figure 3 at the maiden application of DC voltage may provide information on ion mobility $\mu_{i}$ from (3). The calculated ion mobilities $\mu_{i}$ at different temperatures are listed in table 1 . The apparent activation energy concerning the transport phenomena $E_{\mu}$ is obtained by plotting $\log \mu_{i}$ vs $10^{3} / T$ as shown in figure 5 . The value of $E_{\mu}$ which was calculated was found to be $0.80 \mathrm{eV}$. Since we have the DC conductivity of the unpolarized sample (the time dependence of the current in this case is negligible) and the ion mobility $\mu_{i 2}$ it is possible to estimate the mobile ion density $n$ from the following equation:

$$
J=q n \mu E,
$$

the numerically calculated values of $n$ at different temperatures are listed in table 1 .

The present data indicated that the activation energy of the slow phase transformation $\Delta E$ is equal to the transport energy $E_{\mu}$. Therefore, it is believed that the two processes are linked. If this is true, the transformation of the phase should be associated with change in the conduction behaviour. The heating-cooling 
Table 1. The calculated mobility and the number of mobile ion density.

\begin{tabular}{lcc}
\hline Temperature $\left({ }^{\circ} \mathrm{C}\right)$ & $\begin{array}{c}\text { Ion mobility } \\
\mu_{t}\left(10^{-3} \mathrm{~cm}^{2} \mathrm{~V}^{-1} \mathrm{~S}^{-1}\right)\end{array}$ & $\begin{array}{c}\text { Mobile ion density } \\
n\left(10^{16} \mathrm{~cm}^{-3}\right)\end{array}$ \\
\hline 105 & 8.7 & 11.5 \\
110 & 11.5 & 9 \\
115 & 16.1 & 7.29 \\
120 & 19.5 & 6.4 \\
125 & 28.9 & 4.84 \\
\hline
\end{tabular}

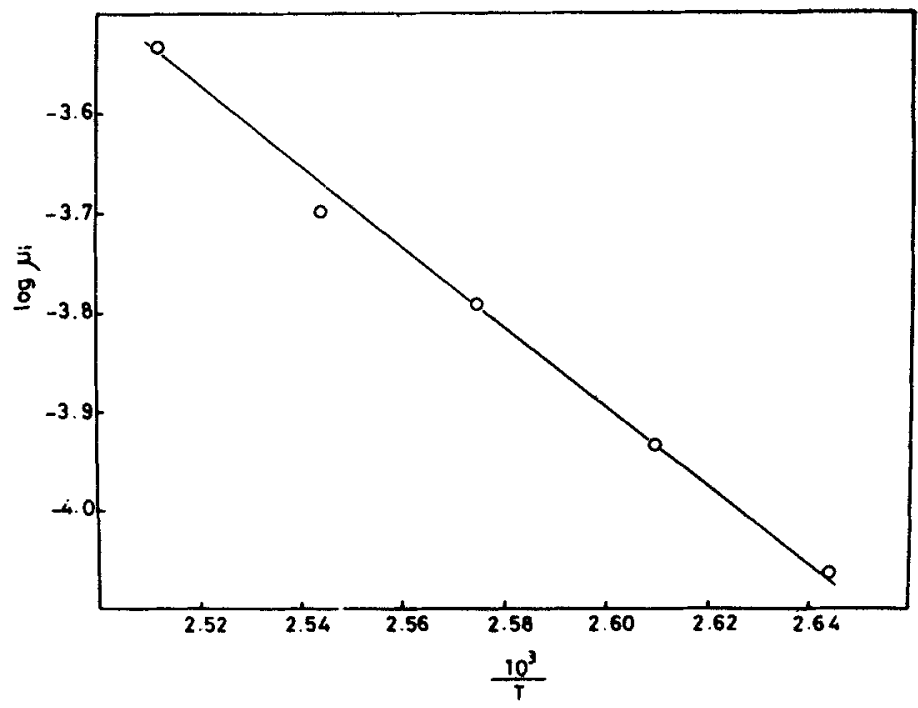

Figure 5. Temperature dependence of mobility $\mu_{i}$ derived from the initial slope of current time curve of figure 3.

process either from the first region to the second region or from the first region to the third region, indicates the decrease of conduction after each cycle as seen in figure 1. Therefore, the phase transformation which is observed above $105^{\circ} \mathrm{C}$ usually leads to decrease in conduction of $\mathrm{Na}(\mathrm{ac}, \mathrm{ac})$. Based on this, it is expected that the conductivity of the reheated sample for the second cycle becomes lower than that of the fresh sample which is experimentally found to be so. However, it was previously reported by using $\mathrm{X}$-ray techniques that $\mathrm{Na}(\mathrm{ac}$, ac) undergoes an irreversible phase transition from orthorhombic to triclinic at temperatures above $82^{\circ} \mathrm{C}$ and the transformation is relatively slow (Kamel et al 1975). Therefore the electrical conductivity results of the present work can be considered as evidence and strong support for the presence of this slow phase transition. On the other hand, the determination of the activation energy of the phase transformation process and the transport energy of the charge carrier indicates the relation between this transformation and the electrical behaviour of $\mathrm{Na}(\mathrm{ac}, \mathrm{ac})$. 


\section{References}

Amin M, Sawaby A and Abd-Ghani S A 1980 Egyptian J. Solids 1(1) 271

Fernelius F C 1946 Inorganic synthesis, (New York: McGraw Hill) vol. 2

Jain V K, Gupta C L and Jain R K 1978 Indian J. Pure Appl. Phys. 16625

Jonscher A K 1967 Thin Solid Films 1213

Kadry N, Sawaby A, El-Agrami A and Sharkawi R 1984 Isotopenpraxis 20312

Kamel R and Attia E A 1961 Acta Met. 91047

Kamel R, Hilal M, Eid A H and Sawaby A 1975 Mol. Cryst. Liq. Cryst. 319

Lowis F H and Sutherland G 1948 J. Org. Chem. 13249

Masamitsu K, Hiroto O and Masayuki L 1970 J. Phys. Soc. Japan 291012

Molokhia N M 1986 Acta Phys. Hung. 60107

Sahai $R$ and Verma R 1981 J. Indian Chem. Soc. 58640

Sawaby A, Afif H H and Shubaka A A 1990 Indian J. Phys. (acceptea) 